\title{
VISUAL RECOVERY UNDER GLARE CONDITIONS FOLLOWING LASER CAPSULOTOMY
}

\author{
MARK WILKINS, ROGER McPHERSON and VERONICA FERGUSSON \\ Camberley
}

\begin{abstract}
SUMMARY
The purpose of this study was to look at the changes in Snellen visual acuity and contrast sensitivity under standard and glare conditions before and after Nd:YAG laser capsulotomy. Contrast sensitivity was measured with a Pelli-Robson chart; a Brightness Acuity Tester was used as a glare source. Seventeen pseudophakic patients with no other ocular disease underwent a capsulotomy. Under standard conditions, 7 showed an improvement in visual acuity and 15 showed an improvement in contrast sensitivity. Under glare conditions, 10 patients showed an improvement in visual acuity and all 17 showed an improvement in contrast sensitivity. The improvement in average contrast sensitivity was greater under glare $(0.330)$ than under standard conditions $(0.210 ; p=0.01)$. Contrast sensitivity measurement, particularly under glare conditions, provides additional information in the assessment of pseudophakes with opaque posterior capsules.
\end{abstract}

Posterior capsule opacification is a known cause of visual disability following cataract surgery. Nd:YAG laser capsulotomy is the standard treatment for this condition. Estimates of the number of patients requiring the procedure vary from $18.4 \%$ to $50 \%$ in patients followed for up to 5 years. ${ }^{1}$ Complications of the procedure include intraocular lens damage, ${ }^{2}$ intraocular pressure rise ${ }^{3}$ cystoid macular oedema ${ }^{4}$ and retinal detachment ${ }^{5}$ it is therefore desirable to avoid unnecessary capsulotomies. Currently the success of a capsulotomy is judged by the improvement in Snellen visual acuity after the procedure.

In patients with cataract, contrast sensitivity is known to be a better index of perceived visual disability than visual acuity. ${ }^{6}$ The combination of glare testing with the Pelli-Robson contrast sensitiv-

From: Frimley Park Hospital, Portsmouth Road, Camberley, Surrey GU16 5UJ, UK.

Correspondence to: Mark Wilkins, FRCOphth, Institute of Ophthalmology, Bath Street, London EC1V 9EL, UK. ity chart $^{7}$ has been shown to be useful in the assessment of patients with cataract. Those patients with denser or clinically significant cataracts had lower scores when glare was produced by shining a pen torch at the pupillary margin. ${ }^{8}$ A comprehensive examination of disability glare tests including the Berkley glare test, and the Pelli-Robson and Regan charts used with a Brightness Acuity Tester showed that the tests provided measures of visual assessment in cataractous and non-cataractous patients that were similarly reliable, discriminative and valid. ${ }^{9}$ Previous studies have looked at the change in visual acuity under glare conditions after laser capsulotomy. ${ }^{10,11}$ In this prospective study Snellen acuity and Pelli-Robson contrast sensitivity were compared under glare and non-glare conditions before and after capsulotomy to determine whether glare conditions and contrast sensitivity testing are helpful in assessing the improvement in quality of vision after Nd:YAG capsulotomy.

\section{METHOD}

Healthy patients with no other ocular disease who had undergone uncomplicated extracapsular cataract surgery with insertion of a posterior chamber implant were included in the study, which took place over a period of 4 months. Those who noticed a subjective post-operative drop in vision were examined by slit lamp biomicroscopy and, if posterior capsule opacification was present, $\mathrm{Nd}$ :YAG laser capsulotomy was recommended.

The best corrected Snellen visual acuity and contrast sensitivity using the Pelli-Robson Letter Sensitivity Chart (Clement Clarke Int. Ltd) were recorded in standard lighting conditions and with glare. The glare came from a Mentor Brightness Acuity Tester (BAT) (Mentor O\&O Inc.), which produces a predetermined, constant bright ambient light. The BAT was used on medium setting to represent glare 'from foliage on a sunny day'. Each 


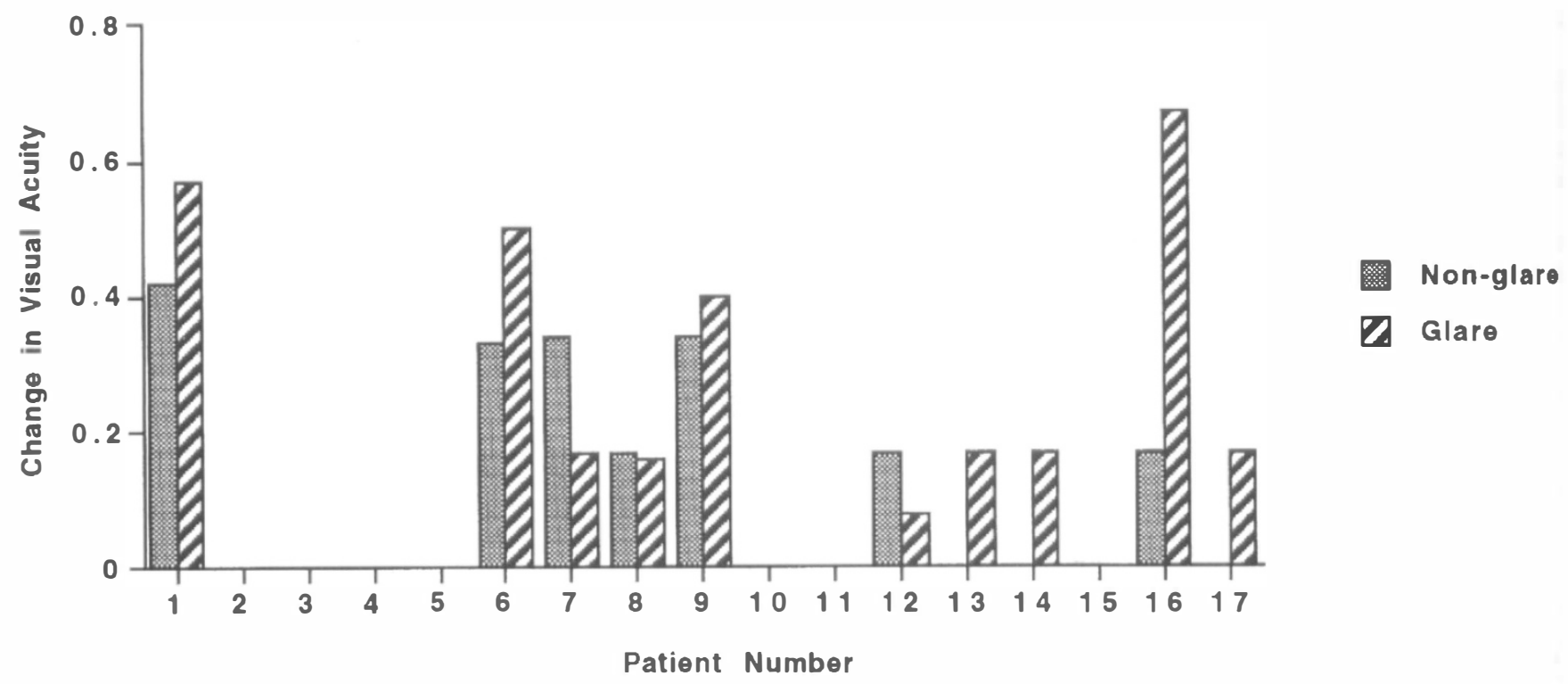

Fig. 1. Changes in visual acuity under glare and non-glare conditions.

patient read the Snellen and Pelli-Robson charts, which were in a room with the same illumination $(90$ $\mathrm{cd} / \mathrm{m}$ ) throughout the study, then each one held the BAT in front of his or her spectacles while reading the charts a second time. The pupils were dilated and capsulotomy was performed with a 'Lasag' Nd:YAG laser. Two weeks later the Snellen acuity and contrast sensitivity were measured as before, with and without the BAT glare source.

The Snellen visual acuities measured before and after capsulotomy were converted to decimal units (6/9 became 0.67). Then both visual acuities and contrast sensitivities were compared statistically using the paired $t$-test, to determine any difference between the means of the two groups, and the sign test to see whether the number of patients showing an improvement in any test category was significant.

\section{RESULTS}

Seventeen patients who had undergone uneventful cataract surgery and subsequently developed posterior capsule opacities between 7 and 265 weeks postoperatively (mean 83 weeks) underwent Nd:YAG laser capsulotomy. The average age was 78 years (range 59-88 years). The results for individual patients are shown Figs. 1 and 2.

Under non-glare conditions the visual acuity improved in 7 patients $(p=0.02$, sign test $)$ and the

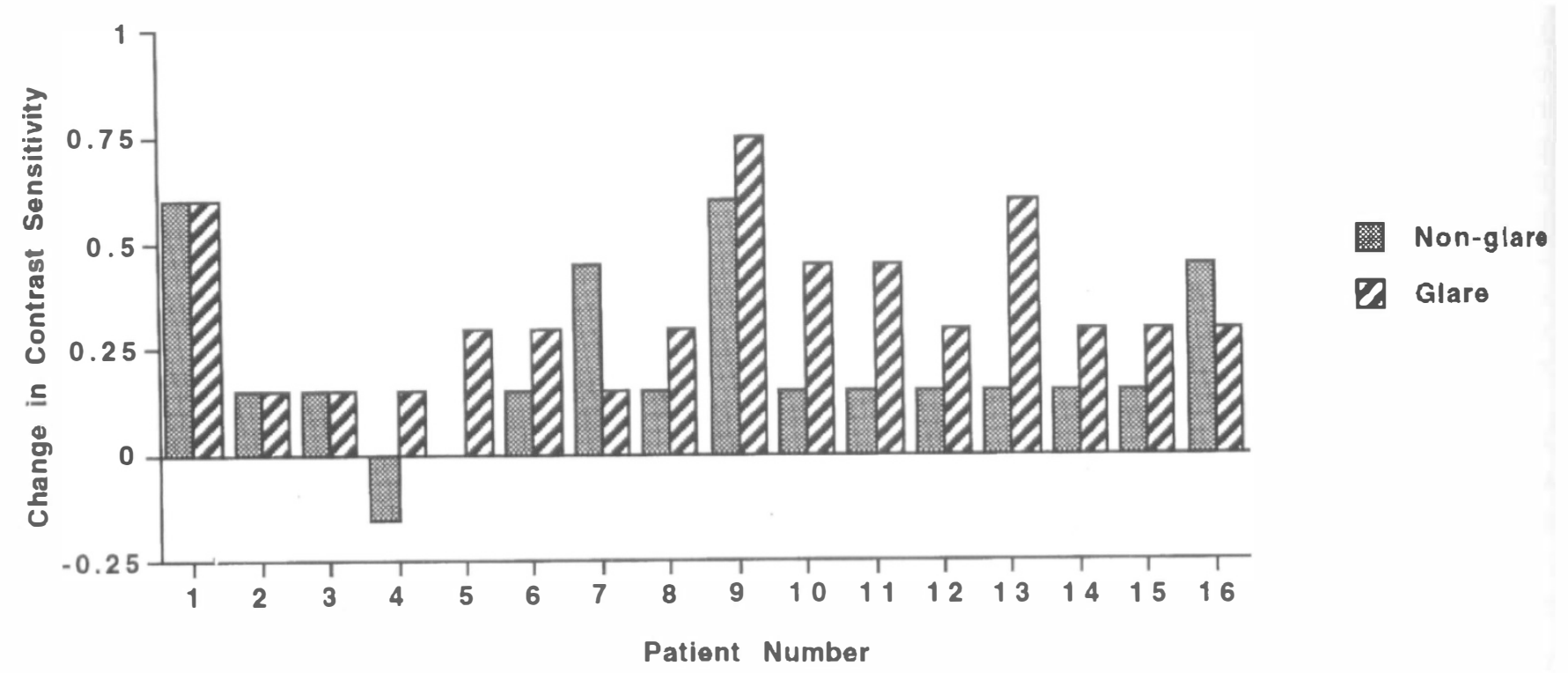

Fig. 2. Changes in contrast sensitivity under glare and non-glare conditions. 
Table I. Average mean changes in visual acuity (VA) and contrast sensitivity (CS) after Nd:YAG laser capsulotomy

\begin{tabular}{|c|c|c|c|}
\hline & Pre-capsulotomy & Post-capsulotomy & Change \\
\hline \multicolumn{4}{|l|}{ Average VA } \\
\hline Non-glare & 0.425 & 0.540 & 0.115 \\
\hline Glare & 0.279 & 0.493 & 0.214 \\
\hline \multicolumn{4}{|l|}{ Average CS } \\
\hline Non-glare & 1.129 & 1.341 & 0.212 \\
\hline Glare & 0.732 & 1.076 & 0.344 \\
\hline
\end{tabular}

contrast sensitivity improved in $15(p=0.0005$, sign test). With the addition of the glare source, 10 patients showed an increase in visual acuity $(p=0.002$, sign test) and all 17 showed an increase in contrast sensitivity $(p<0.0001$, sign test). Seven patients showed no change in visual acuity under glare or non-glare conditions (numbers $2-5,10,11$, 15); all of them showed an improvement in contrast sensitivity under both conditions except for no. 2 (decrease in visual acuity under non-glare conditions) and no. 5 (no change under non-glare conditions). The average improvement in visual acuity under standard conditions was 0.115 , and the average improvement under glare conditions was 0.214 (Table I). The average improvement in contrast sensitivity under standard conditions was 0.212 , and the average improvement under glare conditions was 0.344 . On average the improvement in visual acuity was 0.099 greater when measured under glare conditions $(p=0.08$, paired $t$-test $)$. The improvement in contrast sensitivity was 0.132 better under glare conditions ( $p=0.009$, paired $t$-test).

\section{DISCUSSION}

The results show that on average patients' visual acuity improves under standard non-glare conditions after laser capsulotomy. However, they also demonstrate that the improvement in visual acuity is greater under glare conditions. Some patients appear not to improve under either condition despite having posterior capsule thickening judged to be clinically significant. Looking at contrast sensitivity, most patients show an improvement in contrast sensitivity under non-glare conditions and all show an improvement under glare conditions even when there has been no change in the visual acuity under either condition.

Elliot et al. ${ }^{6}$ showed that there is little correlation between subjective visual disability and monocular or binocular visual acuity measurements in patients with cataract. Binocular contrast sensitivity, however, correlated well with perceived visual disability. Their study also compared subjective visual disability with glare disability (the change in visual acuity or contrast sensitivity produced by glare). However, the glare source used was a BAT which can only be used monocularly. Not surprisingly there was poor correlation between the monocular glare disability scores, which were measured in the worse eye only, and the perceived visual disability scores.

Adamsons' group looked at early cataract and showed that glare and contrast sensitivity measurements are unrelated to visual acuity. ${ }^{12}$ Interestingly, the glare test scores, using sine-wave gratings and a Pelli-Robson chart, were lower in patients with posterior subcapsular lens opacities than in those with nuclear cataract. The Pelli-Robson chart is simple to use but provides a reliable measure of visual assessment in patients with cataracts and compares well with other letter-based charts such as the Regan and Berkley tests. ${ }^{9}$ A simpler glare test using a pen torch and the Pelli-Robson chart has also been successfully used to assess patients with cataract. $^{8}$

Less work has been done on patients who have already had cataract extractions. Ninety-seven per cent of pseudophakic patients show an improvement in visual acuity when measured under glare conditions, compared with $65 \%$ patients showing an improvement when visual acuity is measured under standard conditions. ${ }^{11}$

In our study only 10 of 17 patients showed an increase in visual acuity under glare conditions; all 17 improved their contrast sensitivity scores under glare conditions. The difference between our results and those of Sunderraj et al. ${ }^{11}$ may be due to (1) different glare sources or (2) patient selection. Sunderraj et al. used an Allergan Humphrey 570 autorefractor and we used a BAT. Also, while we observed a thickened posterior capsule at the slit lamp and recommended a laser capsulotomy, in Sunderraj's study the patients presented with complaints about glare. The average visual acuity of our patients pre-capsulotomy was 0.425, whereas in Sunderraj's study it was better, at 0.56 . In our study the patient did not need to complain of glare for a capsulotomy to be performed.

The use of a Pelli-Robson chart combined with a glare source reveals a higher incidence of improved visual function after Nd:YAG capsulotomy than does simply measuring standard Snellen visual acuity or visual acuity under glare conditions. When considering laser capsulotomy one should measure contrast sensitivity, preferably under glare conditions. If the score is poor then a capsulotomy is indicated; if not, then one needs to look for other reasons for poor vision, such as macular disease. 
Key words: Capsulotomy, Contrast sensitivity, Glare, Laser.

\section{REFERENCES}

1. Sterling S, Wood TO. Effect of intraocular lens convexity on posterior capsule opacification. J Cataract Refract Surg 1986;12:655-7.

2. Bath PE, Hoffer KJ, Aron-Rosa D, Dang Y. Glare disability secondary to YAG laser intraocular lens damage. J Cataract Refract Surg 1987;13:309-13.

3. Richter CU, Arzeno G, Pappas HR, Steinert RF, Puliafito C, Epstein DL. Intraocular pressure elevation following Nd:YAG laser posterior capsulotomy. Ophthalmology 1985;92:636-40.

4. Lewis H, Singer TR, Hanscom TA, Straatsma BR. A prospective study of cystoid macular edema after neodymium:YAG laser posterior capsulotomy. Ophthalmology 1987;94:478-82.

5. Leff SR, Welch JC, Tasman W. Rhegmatogenous retinal detachment after YAG laser posterior capsulotomy. Ophthalmology 1987;94:1222-5.

6. Elliott DB, Hurst MA, Weatherill J. Comparing clinical tests of visual function in cataract with the patient's perceived visual disability. Eye 1990;4:712-7.
7. Pelli DG, Robson JG, Wilkins AJ. The design of a new letter chart for measuring contrast sensitivity. Clin Vis Sci 1988;2:187-9.

8. Williamson TH, Strong NP, Sparrow J, Aggarwal RK, Harrad R. Contrast sensitivity and glare in cataract using the Pelli-Robson chart. Br J Ophthalmol 1992; 76:719-22.

9. Elliott DB, Bullimore MA. Assessing the reliability, discriminative ability, and validity of disability glare tests. Invest Ophthalmol Vis Sci 1993;34:108-19.

10. Knighton RW, Slomovic AR, Parrish RKD. Glare measurements before and after neodymium-YAG laser posterior capsulotomy. Am J Ophthalmol 1985;100: 708-13.

11. Sunderraj P, Villada JR, Joyce PW, Watson A. Glare testing in pseudophakes with posterior capsule opacification. Eye 1992;6:411-3.

12. Adamsons I, Rubin GS, Vitale S, Taylor HR, Stark WJ. The effect of early cataracts on glare and contrast sensitivity: a pilot study [see comments]. Arch Ophthalmol 1992;110:1081-6. 